\title{
Development Program to Estimate the Suitable of Raw Materials to Produce Cement
}

\author{
Prof. Dr. Mohammed Mosleh Salman Asmaa Mahdi Ali \\ Civil Engineering Department, College of Engineering, University of Mustansiriayah, Baghdad, Iraq
}

\begin{abstract}
Raw material composition plays an essential role on the lining life of cement rotary kiln. They are obtained from hard rock quarries that represent the first step in the cement manufacturing process. That raw materials are transported to Al- Kufa cement plant then crushed and ground to very fine powder and then blended in the correct proportions. This research aims to study suitable rations of raw materials to produce cement in al Kufa cement plant in Iraq. Through a software program, suitale raw materials ratios for the clinker were estimated, then chemical and physical tests for clinker and cement according to Iraqi Standard Specification were done to recognize the effects on the properties of cement such as the ratios of major and minor oxides, Lime saturations factor (LSF), Silica Modulus (SM) Alumina Modulus (AM), compressive strength, setting time and soundness. Keywords: Portland Cement, Raw material, Cement industry, Rotary kiln and Factors Affective of clinker DOI: $10.7176 / \mathrm{CER} / 11-1-06$
\end{abstract}

\section{Introduction}

Portland cement is a gray non- metallic, inorganic powder, the result of a mixture of calcium silicate (mainly limestone) and aluminoferrites. When cement is mixed with water it forms a paste that sets and hardens due to the formation of calcium silicate hydrates and or calcium aluminates hydrates as a result of the exothermic reaction (RailiKajasteand MarkkuHurme, 2016; - L. M. Aksel'rod, I. G. Maryasev and A. A. Platonov, 2013 )

The raw materials used in the manufacture of cement are limestone, clay, gypsum, slag and pozzolanic materials. The basic stages of cement manufacturing include limestone quarrying, the preparation and pyroprocessing of the raw meal (clinkerization), clinker cooling and cement grinding. The chemical process of cement manufacturing can be described as a calcinations process that initiates with a decomposition of calcium carbonate $\left(\mathrm{CaCO}_{3}\right)$ at about $900^{\circ} \mathrm{C}$ to produce calcium oxide $(\mathrm{CaO}$, lime) with subsequent liberation of carbon dioxide $\left(\mathrm{CO}_{2}\right)$. Following, the clinkering process is conducted in the rotary kiln by reacting calcium oxide at a high temperature $\left(1400-1500^{\circ} \mathrm{C}\right)$ with silica, alumina and ferrous oxide to form the silicates, aluminates and ferrites of calcium which are tha main component of clinker. The clinker cooled and is milled along with gypsum and other additives to produce the gray powder known as cement (Adem Atmaca and RecepYumrutas,2014; Azad Rahman, M.G. Rasul, and S. Sharma,2016)

A cement rotary kiln is a distributed parameter process system which has a highly complex behavior due to chemical reactions. Raw meal for cement production is a mixture of predetermined proportions of limestone, silica and small quantities of alumina and iron oxide. The primary function of a rotary kiln is to provide a hightemperature environment to drive solid- soild and solid-liquid reactions for clinker formation. In the beginning section of a kiln, starting from solid entrance, the calcination of raw material is completed. Thereafter, the soilds undergo soild-soild reactions as they move forward. Then, the solid enters a higher temperature zone where it melts to form a liquid phase. The final reactions take place in this phase. So that it causes formation of coating over the refractory lining in the remaining part of the kiln. In this higher temperature section, called burning zone, the refractory of the kiln is under severe damage because of high temperature. Moreover, the high-coating thickness creates serious problem for the flow of soilds along the kiln. Therefore, the thickness of the coating in the buring zone is momentous because of the protection of the refractory lining from damaging which increases the life of the refractory and movement of feed through the kiln ( Azad Rahman, M.G. Rasul, and S. Sharma,2016; Junli Zhang, Jianguo Liu and Cheng Yiying,2009; A.M. Castañón, S. García-Granda, and M.P. Lorenzo, 2015) Methods based on measuring the thermal radiation from the shell surface were conventional for checking and estimating the coating thickness and lining in the rotary cement kilns (M. Pisaroni, R. Sadi and D. Lahaye,2012)

\subsection{Factors Affecting Burnability:}

The following are the important parameters which affect the burnability of araw mix (Guoxiang Yin,2012; José Luis Aguirre González, 2005; Jacek Szczerba,2010)

\section{a.Chemical Composition:}

Each component of the raw mix has individual and combined [(Lime SaturationFactor (LSF), Silica Modulus (SM), Alumina Modulus (AM),] effects on burnability. The formula, limiting range and the preferable range of the LSF, SM and AM is shown in Table1 


\section{b. Thermal Treatment:}

Burning of the raw mix is generally carried out at $1450-1500^{\circ} \mathrm{C}$. Excessive high burning temperature results in a great stress on the kiln and the refractory lining, more fuel consumption,reduction in cement strength and larger alite crystal .

On increasing holding time, $\mathrm{C}_{3} \mathrm{~A}$ content may decrease, $\mathrm{C}_{4} \mathrm{AF}$ may increase, $\mathrm{C}_{2} \mathrm{~S}$ may decrease, $\mathrm{C}_{3} \mathrm{~S}$ may increase. Rapid burning is always favored as because fine grains of $\mathrm{C}_{2} \mathrm{~S}$ formed which accelerate theinteraction of $\mathrm{C}_{2} \mathrm{~S}$, CaOand liquid. Thermal activation can be enhanced by either with mechanical (vibratory mill) or chemical (mineralizer) activation. Mechanical activation gives better results than chemical.

Table 1: The Formula, Limiting Range And The Preferable Range Of The LSF, SM And AM

\begin{tabular}{|c|c|c|c|}
\hline Parameter & Formula & Limiting range & Preferable range \\
\hline LSF & $\frac{\% \mathrm{CaO}}{28 x \% 5 \mathrm{O} 2 \mathrm{2}+1.2 x \% A 1203+0.65 x \% \text { Fe2O3 }}$ & $0.66-1.02$ & $0.92-0.96$ \\
\hline SM & $\frac{\% 65 i 02}{\% A 1203+\% F_{0203}}$ & $1.9-3.2$ & $2.3-2.7$ \\
\hline $\mathrm{AM}$ & $\frac{\% A 203}{\% F e 203}$ & $1.5-2.5$ & $1.3-1.6$ \\
\hline
\end{tabular}

c. Liquid Phase Formation:

The amount of liquidformed, its appearance temperature, viscosity, surface tension and ionic mobility in the clinkerization process.

\section{d. Clinker Quality:}

The burnability becomes worse as $\mathrm{C}_{3} \mathrm{~S}$ content increases while an increasing $\mathrm{C}_{3} \mathrm{~A}$ and $\mathrm{C}_{4} \mathrm{AF}$, the burnability improves.

e. Kiln Atmosphere:

A reducing atmosphere during burning affect the colour of the clinker by reducing iron oxide,enhancing $\mathrm{C}_{3} \mathrm{~A}$, decreasing $\mathrm{C}_{4} \mathrm{AF}, \mathrm{C}_{3} \mathrm{~S}$.

\section{Experimental Materials:}

Field visits were implemented during 2016 to Al-Kufa Cement plant in Iraq to view the rotary kiln of cement manufacturing. Chemical and physical tests for clinker and cement according to Iraqi Standard Specification were done to recognize the effects on the properties of cement. A software program was achieved to calculate the ratios of the raw materials of clinker.

\section{Results and Discussion}

\subsection{Calculations of Mixing Ratios For Raw Materials:}

A software program is achieved to calculate the mixing ratios of raw materials for the production of the final mixture of the resistant clinker and this program requires:

1- Chemical analysis tests for the raw materials, shown in table 2 that represents the chemical tests of the raw materials.

Table 2: Chemical Tests of Raw Material

\begin{tabular}{|l|c|c|c|c|}
\hline \multicolumn{1}{|c|}{ Compound } & Limestone & Clay & Sand & Iron ore \\
\hline $\mathrm{SiO} 2$ & 2.52 & 39.06 & 87.91 & 16.90 \\
\hline $\mathrm{Al} 2 \mathrm{O} 3$ & 0.23 & 11.42 & 3.14 & 4.05 \\
\hline $\mathrm{Fe} 2 \mathrm{O} 3$ & 0.56 & 7.60 & 1.88 & 62.20 \\
\hline $\mathrm{CaO}$ & 53.12 & 15.35 & 3.24 & 2.48 \\
\hline $\mathrm{MgO}$ & 0.64 & 5.39 & 0.86 & 1.38 \\
\hline $\mathrm{SO} 3$ & 0.64 & 1.59 & 1.22 & 0.50 \\
\hline L.O.I & 41.43 & 17.52 & 1.36 & 10.94 \\
\hline Total & 99.14 & 97.83 & 99.61 & 98.45 \\
\hline
\end{tabular}

2- $\quad$ Specifying the target of $\mathrm{LSF}=0.92, \mathrm{AM}=0.75$ and $\mathrm{SM}=2.6$.

3- Calculating the $\mathrm{CaO}$ of the raw materials under this equation 
$\mathrm{CaO} \max =2.8 * \mathrm{SiO}_{2}+1.2 * \mathrm{Al}_{2} \mathrm{O}_{3}+0.56 * \mathrm{Fe}_{2} \mathrm{O}_{3}$

According to Table 2 :

$\mathrm{C} 1$ of lime stone $=\mathrm{CaO}$ of lime stone $-\left(\mathrm{LSF}^{*} \mathrm{CaOmax}\right.$ of lime stone $)$

$\mathrm{C} 1=46.04$

$\mathrm{C} 2$ of clay $=\mathrm{CaO}$ of clay $-\left(\mathrm{LSF}^{*} \mathrm{CaOmax}\right.$ of clay $)$

$\mathrm{C} 2=128.012$

$\mathrm{C} 3$ of sand $=\mathrm{CaO}$ of sand $-\left(\mathrm{LSF}^{*} \mathrm{CaOmax}\right.$ of sand $)$

$\mathrm{C} 3=227.81$

$\mathrm{C} 4$ of Iron $=\mathrm{CaO}$ of Iron - (LSF* $\mathrm{CaOmax}$ of Iron)

$\mathrm{C} 4=82.721$

Calculation of percentage of primary materials

\section{Mix 1}

$\% \mathrm{C} 2=\mathrm{C} 1 / \mathrm{C} 1+\mathrm{C} 2, \% \mathrm{C} 2$

$\% \mathrm{C} 2=46.039 / 43.039+128.012=0.31$ of clay

$[\% \mathrm{C} 1=1-0.31=0.69$ of limestone $]$

\section{Mix 2}

$\% \mathrm{C} 1=\mathrm{C} 3 / \mathrm{C} 1+\mathrm{C} 3$

$\% \mathrm{C} 1=227.81 / 46.039+227.81=0.83$ of limestone

$\% \mathrm{C} 3=1-0.83=0.168$ of sand

Mix3

$\% \mathrm{Cl}=\mathrm{C} 4 / \mathrm{C} 1+\mathrm{C} 4$

$\% \mathrm{Cl}=82.721 / 82.721+46.039=0.64$ of limestone

$\% \mathrm{C} 4=1-0.64=0.36$ Iron ore

4- Calculating of the main oxides of mixtures:

\section{Mix 1}

Limestone + clay $0.69+0.31$

$\mathrm{SiO}_{2}=0.69 * 2.52+0.31 * 39.06=13.85$

$\mathrm{Al}_{2} \mathrm{O}_{3}=0.69 * 0.23+0.31 * 11.42=3.7$

$\mathrm{Fe}_{2} \mathrm{O}_{3}=0.69 * 0.56+0.31 * 7.6=2.74$

$\mathrm{CaO}=0.69 * 53.12+0.31 * 15.35=41.41$

\section{Mix2}

Limestone + Sand

$$
0.83+0.168
$$

$\mathrm{SiO}_{2}=0.83 * 2.52+0.168 * 87.91=16.88$

$\mathrm{Al}_{2} \mathrm{O}_{3}=0.83 * 0.23+0.168 * 3.14=0.72$

$\mathrm{Fe}_{2} \mathrm{O}_{3}=0.83 * 0.56+0.168 * 1.88=0.78$

$\mathrm{CaO}=0.83 * 53.12+0.168 * 3.24=44.73$

\section{Mix 3}

Limestone+Iron ore

$$
0.64+0.36
$$

$\mathrm{SiO}_{2}=0.64 * 2.52+0.36 * 16.9=7.66$

$\mathrm{Al}_{2} \mathrm{O}_{3}=0.64 * 0.23+0.36 * 4.05=1.60$

$\mathrm{Fe}_{2} \mathrm{O}_{3}=0.64 * 0.56+0.36 * 62.20=22.60$

$\mathrm{CaO}=0.64 * 53.12+0.36 * 2.48=35.01$

From the mixtures above we get mixtures 4 and 5:

Determination of alumina coefficient $(\mathrm{AM})=0.75$

$\mathrm{AM}=\mathrm{Al}_{2} \mathrm{O}_{3} / \mathrm{Fe}_{2} \mathrm{O}_{3}$

$\mathrm{Al}_{2} \mathrm{O}_{3}=0.75^{*} \mathrm{Fe}_{2} \mathrm{O}_{3}$

For Mix 1 (limestone+Clay)

$\mathrm{A} 1=\mathrm{Al}_{2} \mathrm{O}_{3}-0.75 * \mathrm{Fe}_{2} \mathrm{O}_{3}$

$\mathrm{A} 1=3.7-0.75 * 2.74=1.64$

For Mix 2 (limestone+sand)

$\mathrm{A} 2=0.72-0.75 * 0.78=0.132$

For Mix 3 (limestone+Iron ore)

A3 $=1.6-0.75 * 22.6=15.36$

Calculation of percentage of primary materials 


\section{Mix 1}

$\% \mathrm{~A} 3=\mathrm{A} 3 / \mathrm{A} 1+\mathrm{A} 3$

$\% \mathrm{~A} 3=15.36 / 1.64+15.36=0.9035 \%$ of limestone + clay

$1-0.9035=0.096 \%$ limestone+Iron

\section{$\underline{\text { Mix } 3}$}

$\% \mathrm{~A} 2=\mathrm{A} 2 / \mathrm{A} 2+\mathrm{A} 3$

$\%$ A $2=15.35 / 15.35+0.1327=0.9914 \%$ of limestone + sand

$1-0.9914=0.0087 \%$ limestone + Iron

of the above ratio, the main oxides can be calculated by mixing No.4 and

\section{Mix 4}

$\mathrm{SiO}_{2}=0.9035 * 13.85+0.096 * 7.66=13.85$

$\mathrm{Al}_{2} \mathrm{O}_{3}=0.9035 * 3.70+0.096 * 1.6=3.5$

$\mathrm{Fe}_{2} \mathrm{O}_{3}=0.9035 * 2.74+0.096 * 22.6=4.66$

$\mathrm{CaO}=0.9035 * 41.41+0.096 * 35.01=40.79$

$\underline{\operatorname{Mix} 5}$

$\overline{\mathrm{SiO}_{2}}=0.991 * 16.88+0.008 * 7.66=16.80$

$\mathrm{Al}_{2} \mathrm{O}_{3}=0.991 * 0.72+0.008 * 1.6=0.73$

$\mathrm{Fe}_{2} \mathrm{O}_{3}=0.991 * 0.78+0.008 * 22.6=0.97$

$\mathrm{CaO}=0.991 * 44.73+0.008 * 35.01=44.65$

To calculate the final mixture for the production of the resistant clinker by combining mixing No.4 and 5 by setting the coefficient of silica to be 2.6

$\mathrm{SM}=\mathrm{SiO}_{2} /\left(\mathrm{Al}_{2} \mathrm{O}_{3}+\mathrm{Fe}_{2} \mathrm{O}_{3}\right)$

$\mathrm{S}=\mathrm{SiO}_{2}-\mathrm{SM}\left(\mathrm{Al}_{2} \mathrm{O}_{3}+\mathrm{Fe}_{2} \mathrm{O}_{3}\right)$

$\mathrm{S} 4=13.25-[2.6(3.5+4.66)]=-7.96$

$\mathrm{S} 5=16.8-[2.6(0.73+0.97)]=12.39$

$\% \mathrm{~S} 5=\mathrm{S} 4 / \mathrm{S} 5+\mathrm{S} 4$

$\% \mathrm{~S} 5=7.96 /(7.96+12.39)=0.3912 \%$ of $[($ lime + Iron $)+($ lime + sand $)]$

$\%$ S4 $=1-0.3912=0.6088 \%$ of $[($ lime + clay $)+($ lime + iron $)]$

\section{Mix 6}

$\mathrm{SiO}_{2}=0.3912 * 16.80+0.61 * 13.25=14.639$

$\mathrm{Al}_{2} \mathrm{O}_{3}=0.3912 * 0.73+0.61 * 3.5=2.41$

$\mathrm{Fe}_{2} \mathrm{O}_{3}=0.3912 * 0.97+0.61 * 4.66=3.22$

$\mathrm{CaO}=0.3912 * 44.65+0.61 * 40.79=42.30$

The percentages of the mixing of the raw materials in the process of production of the Al-Kufa Cement plant in Iraq during 2016 is listed in tables below using the software program.

Table 3: Final Chemical Tests of Raw Material

\begin{tabular}{|l|c|c|c|c|}
\hline \multicolumn{1}{|c|}{ Compound } & Limestone & Clay & Sand & Iron ore \\
\hline $\mathrm{SiO} 2$ & 2.36 & 40.64 & 88.02 & 2.65 \\
\hline $\mathrm{Al} 2 \mathrm{O} 3$ & 0.26 & 13.89 & 4.22 & 0.9 \\
\hline $\mathrm{Fe} 2 \mathrm{O} 3$ & 0.48 & 6.08 & 0.70 & 92.64 \\
\hline $\mathrm{CaO}$ & 51.82 & 15.87 & 1.62 & 2.31 \\
\hline $\mathrm{L} . \mathrm{O} . \mathrm{I}$ & 42.97 & 15.66 & 3.35 & 0.9 \\
\hline
\end{tabular}

Table 4: Ratios Of Raw Materials For Clinker

\begin{tabular}{|l|c|c|c|}
\hline \multicolumn{1}{|c|}{ Compound } & Mix1 & Mix2 & Mix3 \\
\hline $\mathrm{SiO} 2$ & 13.89 & 2.48 & 16.402 \\
\hline $\mathrm{A} 2 \mathrm{O} 3$ & 4.37 & 0.52 & 0.909 \\
\hline $\mathrm{Fe} 2 \mathrm{O} 3$ & 2.17 & 37.81 & 0.516 \\
\hline $\mathrm{CaO}$ Compound & 40.99 & 31.77 & 43.591 \\
\hline $\mathrm{SiO} 2 \quad$ Mix4 & Mix 5 & Mix 6 \\
\hline $\mathrm{A} 2 \mathrm{O} 3$ & 13.02 & 16.16 & 14.277 \\
\hline $\mathrm{Fe} 2 \mathrm{O} 3$ & 4.07 & 0.90 & 2.801 \\
\hline $\mathrm{CaO}$ & 4.91 & 1.17 & 3.406 \\
\hline
\end{tabular}


Table5: Raw Materials For Clinker

\begin{tabular}{|l|c|}
\hline \multicolumn{1}{|c|}{ Compound } & Clinker \\
\hline $\mathrm{SiO} 2$ & 22.27 \\
\hline $\mathrm{A} 12 \mathrm{O} 3$ & 4.37 \\
\hline $\mathrm{Fe} 2 \mathrm{O} 3$ & 5.31 \\
\hline $\mathrm{CaO}$ & 64.78 \\
\hline L.S.F & 0.912 \\
\hline
\end{tabular}

Table 6: Ratios Of Raw Materials For Clinker

\begin{tabular}{|l|l|l|}
\hline \multirow{2}{*}{ Mix 1 } & \% limestone & 0.70 \\
\cline { 2 - 3 } & \% clay & 0.30 \\
\hline \multirow{2}{*}{ Mix2 } & \%Limestone & 0.84 \\
\cline { 2 - 3 } & \% sand & 0.16 \\
\hline \multirow{2}{*}{ Mix3 } & \%limestone & 0.595 \\
\cline { 2 - 3 } & \%iron & 0.405 \\
\hline \multirow{2}{*}{ Mix4 } & \%lime+clay & 0.92 \\
\cline { 2 - 3 } & \% lime+iron & 0.08 \\
\hline \multirow{2}{*}{ Mix5 } & \%lime+sand & 0.983 \\
\cline { 2 - 3 } & \%lime+iron & 0.017 \\
\hline \multirow{2}{*}{ Mix6 } & \% lime+iron+clay+sand & 0.401 \\
\cline { 2 - 3 } & \% lime+clay & 0.599 \\
\hline
\end{tabular}

Table7: Final Ratio Of Raw Material For Clinker

\begin{tabular}{|c|c|}
\hline Limestone & 0.747 \\
\hline Clay & 0.167 \\
\hline Iron & 0.021 \\
\hline sand & 0.065 \\
\hline
\end{tabular}

\subsection{Physical And Chemical Tests Of Clinker And Cement:}

Samples were taken for clinker and cement produced in the Al-Kufa cement plant in Iraq during 2016. Below are the results chemical and physical tests according to Iraqi Standard Specification. 


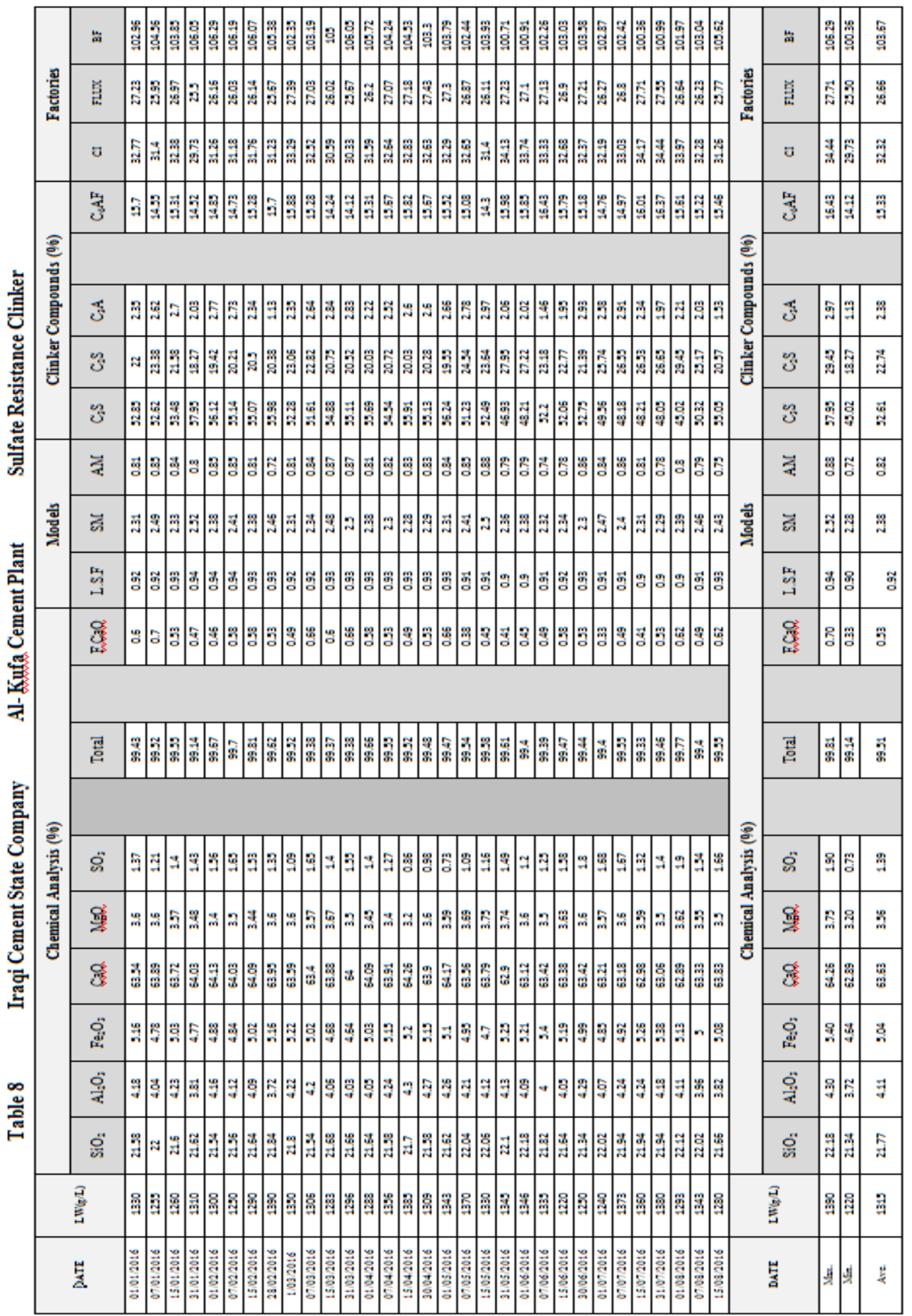




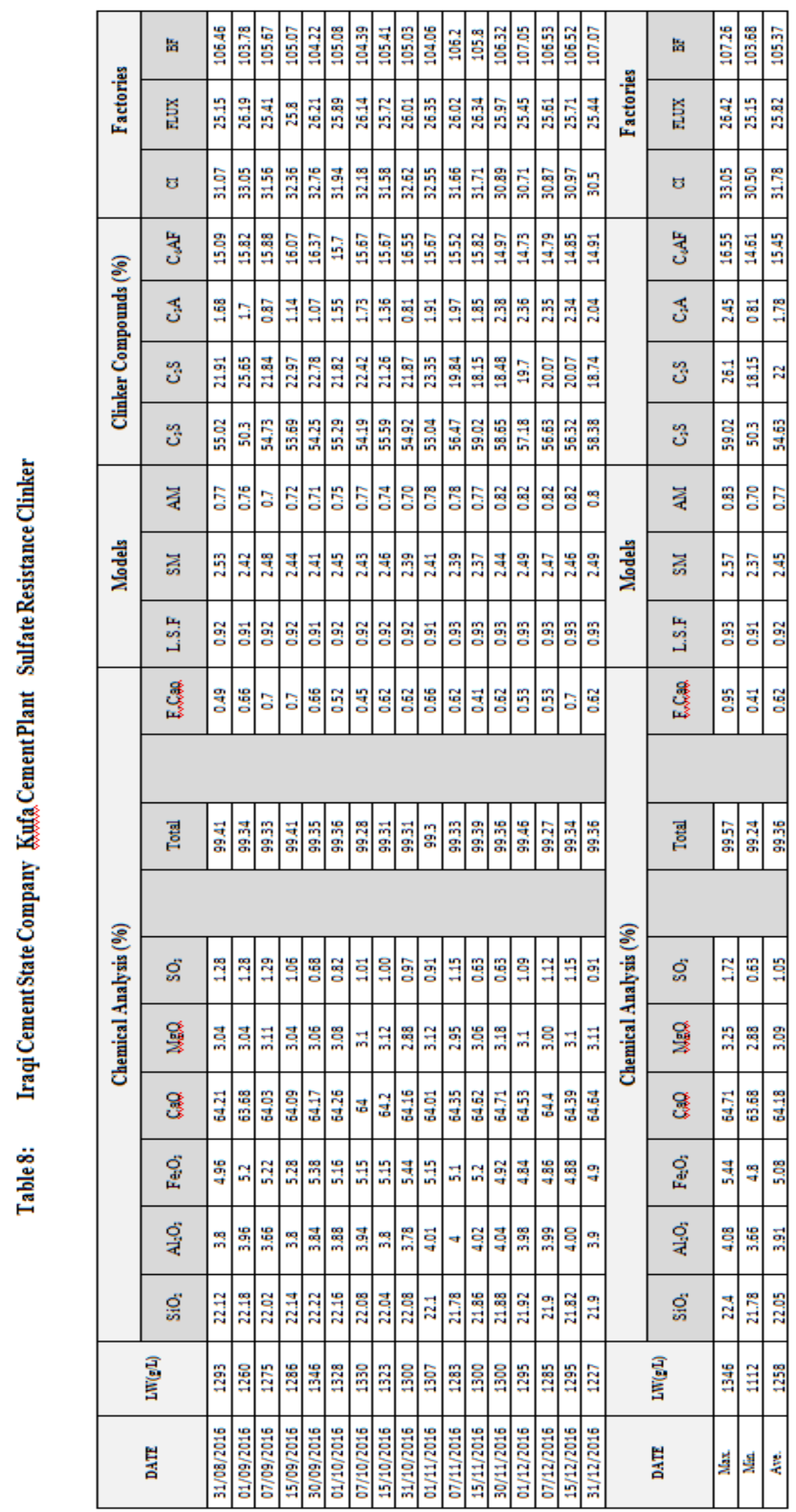




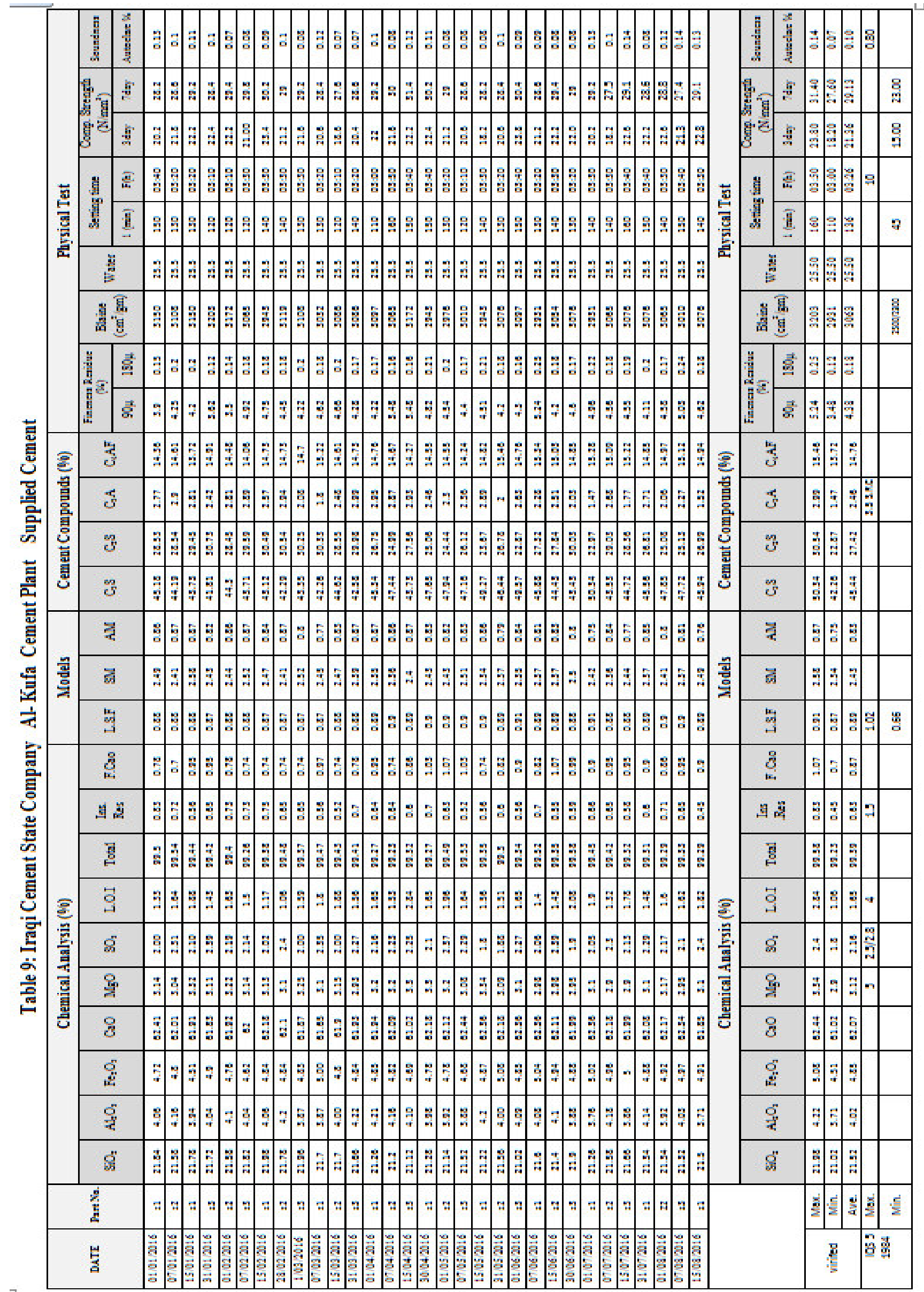




\begin{tabular}{|c|c|c|c|c|c|c|c|c|c|c|c|c|c|c|c|c|c|c|c|c|c|c|c|c|c|c|}
\hline \multirow{9}{*}{ 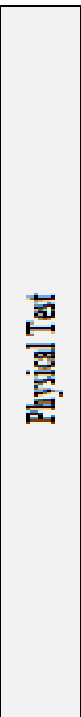 } & $\begin{array}{l}\text { 吾 } \\
\text { 咅 }\end{array}$ & $\frac{\pi}{4}$ & : & $\mathrm{g}$ & z & a & & 畕 & $\mathrm{g}$ & $a$ & 兽 & 열 & $\mathrm{g}$ & $\mathrm{g}$ & $B$ & z & $\mathrm{g}$ & & $\mathrm{g}$ & \multirow{9}{*}{ 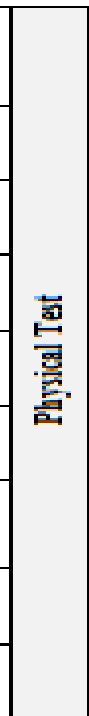 } & I & & $\mathrm{g}$ & 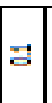 & $\mathrm{g}$ & \\
\hline & $\mathrm{F}$ & $\mathrm{E}$ & $\approx$ & 9 & $\pi$ & s & 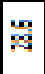 & $=$ & $\pi$ & $=$ & $\exists$ & 7 & 9 & 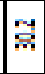 & $\approx$ & 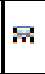 & $\%$ & $\exists$ & $\pi$ & & it: & & 9 & 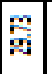 & & $\mathrm{B}$ \\
\hline & $g^{2}$ & i & $\mathrm{s}$ & 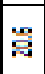 & $\exists$ & $\mathrm{a}$ & $g$ & $\bar{s}$ & $\mathrm{a}$ & 目 & 9 & $\approx$ & a & $\mathrm{z}$ & $m$ & 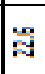 & a & $\mathrm{A}$ & $\vec{a}$ & & $g$ & $\vec{z}$ & 苛 & $\mathrm{g}$ & & 9 \\
\hline & \multirow{2}{*}{ 武 } & 要: & 8 & 8 & 莌 & 궁 & 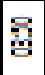 & 궁 & 8 & : & 8 & 8 & 8 & 3 & 量 & 9 & 8 & 孚 & 굴 & & \multirow{2}{*}{ I } & 3 & 굴 & 9 & $\Rightarrow$ & \\
\hline & & 五 & 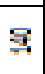 & 注 & $: 9$ & : & 政 & ; & I & 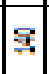 & F & 9 & g & 园 & : & g & I & $\mathrm{g}$ & $\mathrm{a}$ & & & : : & 园 & 5 & & $\Rightarrow$ \\
\hline & & $\mathrm{a}$ & 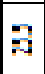 & $\mathrm{a}$ & $a$ & $\mathrm{a}$ & $\mathrm{a}$ & 3 & 3 & $a$ & 3 & $a$ & 2 & $a$ & $a$ & 9 & 9 & 9 & & 2 & 宗 & 3 & 3 & & \\
\hline & \multicolumn{2}{|c|}{ 獣晋 } & 急 & 8 & 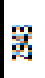 & s & $\mathrm{s}$ & 解 & 璂 & 国 & 寻 & 湆 & 浔 & 3 & 寻 & 畄 & 搳 & 灵 & 㶻 & & 高晋 & 酸 & 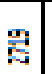 & $\mathrm{g}$ & & 골 \\
\hline & \multirow{2}{*}{ 咅 } & & 영 & $\mathrm{g}$ & $\mathrm{z}$ & 영 & 영 & $\mathrm{g}$ & 영 & 圈 & $\mathrm{g}$ & $\mathrm{g}$ & 罗 & $\mathrm{z}$ & $\mathrm{g}$ & 娄 & 9 & 粤 & 圈 & & \multirow{2}{*}{$\frac{3}{1}=$} & 3 & 영 & 罗 & & \\
\hline & & $\dot{s}$ & 8 & 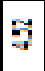 & $\mathrm{O}$ & 9 & 9 & 3 & 9 & 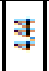 & 3 & $g$ & $\%$ & $\mathrm{~g}$ & 9 & 5 & 3 & $\vec{z}$ & $m$ & & & 9 & $m$ & 9 & & \\
\hline \multirow{4}{*}{ 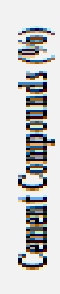 } & \multicolumn{2}{|c|}{ 学 } & $\frac{\bar{g}}{9}$ & 8 & $\exists$ & 3 & $\mathrm{~s}$ & 3 & $\vec{z}$ & 5 & 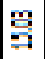 & 3 & 3 & 3 & 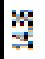 & $\mathrm{s}$ & 9 & $\overrightarrow{3}$ & $\exists$ & \multirow{4}{*}{ 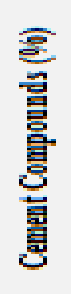 } & 宩 & 5 & 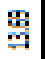 & $\mathrm{g}$ & & \\
\hline & \multicolumn{2}{|c|}{ 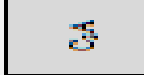 } & $\mathrm{E}$ & 9 & $\exists$ & 寻 & $\mathrm{s}$ & 3 & 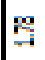 & :우 & 9 & 9 & 23 & $\mathrm{~A}$ & 9 & $\sigma$ & 8 & $z$ & $\mathrm{a}$ & & 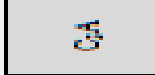 & 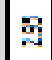 & $\exists$ & 9 & 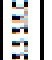 & \\
\hline & \multicolumn{2}{|c|}{3} & 弯 & $\mathrm{s}$ & a & 圈 & 8 & : & 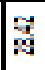 & $\overrightarrow{8}$ & $\overrightarrow{0}$ & $\vec{\beta}$ & 9 & 9 & 哭 & 5 & $g$ & a & 9 & & 3 & 9 & 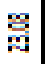 & 空 & & \\
\hline & \multicolumn{2}{|c|}{3} & 急 & 3 & 9 & 9 & 9 & 9 & 9 & 3 & 䓓 & 9 & 掌 & 3 & 窸 & 5 & $\frac{9}{9}$ & 9 & 弯 & & 3 & 窸 & 9 & $\mathrm{~g}$ & & \\
\hline \multirow{3}{*}{ 券 } & 켜 & & $\mathrm{g}$ & $\mathrm{E}$ & $\mathrm{E}$ & 염 & : & 흠 & g & 8 & 3 & 영 & $: 3$ & 3 & 5 & g & 5 & 5 & 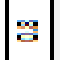 & \multirow{3}{*}{ 㖓 } & 굴 & 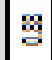 & $\mathrm{B}$ & 영 & & \\
\hline & 政 & & $\mathrm{g}$ & $z$ & 韦 & 5 & : & $\approx$ & 9 & $\approx$ & 9 & 9 & 5 & 要 & $\approx$ & 9 & $: 9$ & $\therefore$ & $\ddot{A}$ & & 충 & 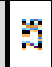 & $\mathrm{P}$ & 乎 & & \\
\hline & 学 & & $\mathrm{s}$ & : & ; & $\mathrm{g}$ & 켬 & : & 圈 & 解 & 涪 & 圈 & 영 & 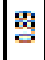 & 혐 & 圈 & 豆 & 영 & $\mathrm{a}$ & & 3 & 형 & 9 & $\mathrm{~g}$ & : & $\mathrm{g}$ \\
\hline & 8 & & 5 & 9 & $\mathrm{~g}$ & $\exists$ & $\exists$ & : & $g$ & : & $\mathrm{a}$ & 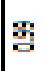 & 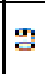 & $\mathrm{m}$ & 9 & $\vec{s}$ & g & : & $\exists$ & & 3 & $\mathrm{~g}$ & है & 9 & & \\
\hline & . & & : & $\mathrm{E}$ & : & $\mathrm{g}$ & 9 & 형 & $\mathrm{a}$ & $=$ & 형 & 9 & $\mathrm{~g}$ & $\mathrm{E}$ & 3 & 9 & $\mathrm{~B}$ & g & 3 & & 멸 & 9 & 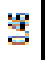 & 형 & $\Rightarrow$ & \\
\hline & $\mathrm{g}$ & & 疍 & 3 & 采 & 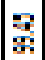 & 3 & $z$ & 橧 & 3 & 8 & 墨 & 9 & 3 & 罾 & 紊 & 9 & 兽 & 8 & & $\mathrm{Z}$ & 3 & 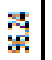 & 9 & & \\
\hline$\overline{3}$ & 9 & & 采 & $\because$ & $\Rightarrow$ & : & $\mathrm{A}$ & $\theta$ & $\therefore$ & $\vec{z}$ & $m$ & 5 & 5 & 3 & 9 & 5 & $: 8$ & $\vec{a}$ & $:$ : & $\bar{\varepsilon}$ & 8 & $\therefore$ & 9 & $A$ & $=$ & \\
\hline 吾. & $\dot{\beta}$ & & 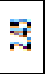 & F & 9 & : & 9 & 9 & $\%$ & $\nexists$ & 9 & $\rightarrow$ & 9 & 9 & 9 & 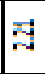 & $\mathrm{g}$ & $a$ & $\mathrm{~g}$ & 毁 & $\ddot{\partial}$ & 类 & : & 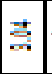 & $\frac{9}{2}$ & \\
\hline . 賀 & 9 & & $\mathrm{~s}$ & 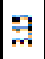 & 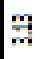 & 9 & 3 & $=$ & $m$ & $\approx$ & $\sigma$ & $m$ & 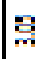 & 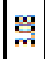 & $\Rightarrow$ & $m$ & 9 & $m$ & $\Rightarrow$ & . & 9 & ; & ; & 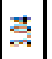 & $\because$ & \\
\hline & 3 & & $\mathrm{z}$ & 3 & 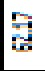 & 9 & 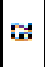 & 丞 & 3 & : & 急 & $\overrightarrow{8}$ & 9 & 3 & 8 & 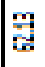 & 5 & 丞 & $\mathrm{s}$ & $\Xi$ & 8 & 3 & 3 & $\overrightarrow{3}$ & & \\
\hline & 3 & & 9 & A & $\exists$ & $\mathrm{s}$ & $\mathrm{z}$ & 9 & $\Rightarrow$ & 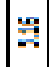 & 3 & 9 & $\Rightarrow$ & 5 & 5 & 9 & 5 & 5 & 3 & & 3 & $\Xi$ & $\Rightarrow$ & $\%$ & & \\
\hline & 열 & & $\sigma_{m}$ & $\mathrm{~g}$ & $\mathrm{~m}$ & $\exists$ & $\exists$ & 9 & 9 & $\mathrm{~s}$ & g & 9 & 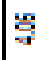 & 3 & $\Rightarrow$ & $\mathscr{g}$ & 9 & 9 & $\mathrm{~B}$ & & 3 & $\exists$ & 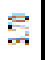 & $\because$ & & \\
\hline & 8. & & $\mathrm{A}$ & 9 & 9 & 곡 & $\pi$ & A & 9 & 굴 & $\vec{*}$ & 3 & 9 & 9 & 品 & 3 & 囬 & 9 & 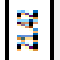 & & 8 & 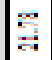 & ३ & 罚 & & \\
\hline & 总 & & $\approx$ & $\approx$ & 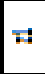 & & $\Rightarrow$ & $\Rightarrow$ & $\Rightarrow$ & $\Rightarrow$ & & $\Leftrightarrow$ & $\approx$ & $\Rightarrow$ & $\Rightarrow$ & $\%$ & $\Rightarrow$ & & $\approx$ & & & 音 & $\div$ & 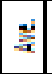 & 童 & $=$ \\
\hline & 봄 & & 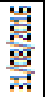 & & & & & & & & & & & 盽 & $\frac{\mathrm{g}}{\mathrm{i}}$ & 홓 & 畓 & $\frac{3}{3}$ & $\frac{3}{3}$ & & & & $\mathrm{z}$ & & 9 & 貫 \\
\hline
\end{tabular}

\section{Conclusions}

1- The basis for this property is well-burned clinker with consistent chemical composition and free lime. There are only two reasons for the clinker free lime to change in a situation with stable kiln 
operation: variation in the chemical composition of the kiln feed or variations in its fineness. Variations in fineness depend on possible changes in raw materials or in operation of the raw mill. Variation in chemical composition is related to raw mix control and the homogenization process.

2- Refractory chemistry is important because it helps determine if a given product is compatible with certain applications. Although the chemical composition alone is not a suitable criterion to define product usage.

3- The finer the raw mix will have to be ground to ensure satisfactory combination at acceptable kiln temperatures. When decarbonation is complete at about $1100 \mathrm{C}$, the feed temperature rises more rapidly. Lime reacts with silica to form belite (C2S) but the level of unreacted lime remains high until a temperature of $\sim 1250 \quad \mathrm{C}$ is reached. This is the lower limit of the rmodynamic stability of alite (C3S). At $\sim 1300 \quad \mathrm{C}$ partial melting occurs, the liquid phase (or flux) being provided by the alumina and iron oxide present. The level of unreacted lime reduces as $\mathrm{C} 2 \mathrm{~S}$ is converted to $\mathrm{C} 3 \mathrm{~S}$. The process will be operated to ensure that the level of unreacted lime (free lime) is below $3 \%$.

4- As the clinker passes under the flame it starts to cool and the molten $\mathrm{C} 3 \mathrm{~A}$ and $\mathrm{C} 4 \mathrm{AF}$, which constitute the flux phase, crystallize. This crystallization is normally complete by the time the clinker exits the rotary kiln and enters the cooler at a temperature of 1200 C. Slow cooling should be avoided as this can result in an increase in the belite content at the expense of alite and also the formation of relatively large C3A crystals which can result in unsatisfactory concrete rheology .

5- Higher amount of sandstone is hard to burn in kiln because it consumes large amount of coaml and it even increases free lime.

6- Percentage of $\mathrm{C}_{3} \mathrm{~S}$ content in clinker in increased. Alite hardens the cement faster and contributes to early strength formation. It resistant to sulfur attack hence high strength of $\mathrm{C}_{3} \mathrm{~S}$ will increase the strength at all ages.

7- Lime saturations factor (LSF), Silica Modulus (SM) and Alumina Modulus (AM) and variations in clinker quality on the limit. It can be decreased by carrying out various steps at different level which reduces the deviations of blending efficiency, raw mill feed, kiln feed and clinker compositions and its minerals.

8- LSF should be in the range of $92 \% \pm 1$, but minimum value obtained is $89 \%$. To determine the silica and the SM minimum value obtained is 2.3 and it goes to a maximum value of 2.65 . The minimum AM value obtained is 0.7 and it goes to a maximum value of 0.9 . The importance of the calculation is preparation a kiln feed mixture with a suitable content of calcium carbonate (61-65\%) to avoid hard burning process at high content of calcium carbonate content, as well as to avoid friction resulting from the silica on the formed coat on the internal kiln wall when calcium carbonate content is low.

9- When the LSF is above 0.95 there is an excess of lime, which cannot be combined no matter how long the clinker is fired and this remains as free lime in the clinker. As a low level of uncombined lime must be achieved the influence of LSF on the content of C3S and C2S.

10- The higher silica modulus (SM) the less molten liquid or flux is formed. This makes clinker combination more difficult unless the LSF is reduced to compensate. The flux phase facilitates the coalescence of the clinker into nodules and also the formation of a protective coating on the refractory kiln lining.

11- High Alumina Modulus (AM) cements will have a high C3A content, and this can be disadvantageous in certain cement applications, where it is desired to minimize the concrete temperature rise.

12- The SO3 have to be closely controlled because they are volatilized in the kiln and can cause severe operational problems associated with their condensation and the formation of build-ups in the kiln.

\section{References}

Raili Kajaste and Markku Hurme " Cement Industry Greenhouse Gas Emissions Management Options And Abatement Cost" Aalto University, Department of Biotechnology and Chemical Technology, , Finland,Journal of Cleaner Production, Vol.112, 2016, pp.4041-4052.

L. M. Aksel'rod, I. G. Maryasev and A. A. Platonov, "Method Of Determining The Resistance Of Furnace Refractories To Slag And Clinker” , Journal of Refractories and Industrial Ceramics Vol. 54, No. 4, 2013, pp. $59-64$.

Adem Atmaca and RecepYumrutas," Analysis Of The Parameters Affecting Energy Consumption Of A RotaryKiln In Cement Industry", University of Gaziantep, Department of Mechanical Engineering, , Turkey, Journal of Applied Thermal Engineering, Vol.66, 2014, pp.435-444.

Azad Rahman, M.G. Rasul, and S. Sharma" Cement Kiln Process Modeling to Achieve Energy Efficiency by Utilizing Agricultural Biomass as Alternative Fuels" School of Engineering and Technology, Australia, 
chapter 9, 2016, pp.197-225.

Junli Zhang, Jianguo Liu and Cheng Yiying" Comparison Of The Fixation Effects Of Heavy Metals By Cement Rotary Kiln"Department of Environmental Science and Engineering, Tsinghua University, China, Journal of Hazardous Materials, Vol. 165, 2009, pp.1179-1185.

A.M. Castañón, S. García-Granda, and M.P. Lorenzo "Energy And Environmental Savings Via Optimisation Of The ProductionProcess At A Spanish Cement Factory" ,Journal of Cleaner Production, Vol. 98 , 2015, pp.47-52.

M. Pisaroni, R. Sadi and D. Lahaye"Counteracting Ring Formation In Rotary Kilns" Journal ofMathematics in Industry,2012,http://www.mathematicsinindustry.com

Guoxiang Yin"High Performance Iron-Rich Magnesia-Spinel Composite For Burning Zone Of Cement Rotary Kiln" School of materials Science and Engineering, University of Science and Technology Beijing,China, Advanced Materials Research , ISSN: 1662-8985, Vols. 476, 2012,, pp 1915-1919.

B.Gerald and M.T.Homas,"Magnesia-Bricks An Innovative Burnt Basic Refractory",inProceedingsofUNITECR'99,Osaka,Japan,2009, pp. 201-203.

G. Gelbmann, R. Krischanitz and S. Joerg Austria "Hybrid Spinels Technology For Basic Bricks In Chemically Highly Loaded Cement Rotary Kilns" Proceedings of the Unified International Technical Conference on Refractories, American Ceramic Society,2014, PP.1-3 